\title{
Interaction Design in the wild
}

\author{
Dorothy Rachovides, David Frohlich \\ Digital World Research Centre \\ University of Surrey \\ Guildford GU2 7XH, UK \\ \{d.rachovides, d.frohlich\}@surrey.ac.uk
}

\author{
Maxine Frank \\ VOICES \\ $9^{\text {th }}$ Cross, $1^{\text {st }}$ Stage, Indiranagar \\ Bangalore, 560038, India \\ maxine.frank@gmail.com
}

\begin{abstract}
The StoryBank project in the UK is exploring the application of digital storytelling technology to information sharing in the developing world. A multidisciplinary team of interaction designers, ethnographers and computer scientists are adopting a user-centered approach to the design of a system which should be useful to a specific rural community in South India. This paper discusses some of the challenges that the interaction designers met and how these shaped the design process.
\end{abstract}

\section{Categories and Subject Descriptors}

H.5.2 [Information Interfaces and Presentation]: User Interfaces - Prototyping, User-centered design, control structures.

\section{General Terms}

Design, Human Factors.

\section{Keywords}

Digital divide, ICT4D.

\section{INTRODUCTION}

The UK Engineering and Physical Sciences Research Council in the UK recently funded four ICT-for-development research projects. All these projects are multidisciplinary with partners in some of the poorest parts of the world. They are strongly committed to a participative design process which leads to a sustainable technology intervention of real value to end users. This paper presents some of the design challenges that the StoryBank project has met and how they have been addressed. This is done through a case study with the work carried out early in 2007.

\section{BACKGROUND}

\subsection{The Location}

StoryBank has partnered with VOICES, a local NGO (NonGovernmental Organization) in Bangalore who provide media infrastructure to facilitate communication within local communities. With UNESCO funding, VOICES in partnership with Myrada (an other NGO) have set up a community radio station, Namma Dhwani ('Our Voices') in the village of Budikote in Kolar. The radio station is hosted in the village ICT (Information and Communication Technologies) resource

(C) Dorothy Rachovides, David Frohlich, Maxine Frank 2007 Published by the British Computer Society

Volume 2 Proceedings of the 21st BCS HCI Group Conference

HCI 2007, 3-7 September 2007, Lancaster University, UK Devina Ramduny-Ellis \& Dorothy Rachovides (Editors) centre, where a variety of programs are made both by staff and the wider community. These radio programs are broadcasted over the cable TV network, or narrow cast, using the village Public Address speakers. The narrow cast extends to two neighboring villages. Radio programs are also used at meetings of self help groups and sold over to other community radio stations in the region. Budikote is on the borders of Karnataka, Andhra Pradesh and Tamil Nadu, and as a consequence a mixture of three local languages (Kannada, Telugu, Tamil) is spoken giving a local dialect. The village population is about 3000 , which is typical of rural Indian villages. It is the head village of the area, so the local government offices, Panchyat, Health Centre, primary and secondary schools are located there.

Due to the radio station which was setup in 2001, the village has attracted interest from westerns and the community is very friendly and helpful. Although the majority of the population is illiterate and many have not traveled further than Bangarpet, which is a 30 minute ride on the bus, they are very open to the use of technology. Despite the limited power supply, 2 hours a day, most homes have TV sets. Some people have mobile phones, but until recently the village had no mobile phone coverage, so this was a luxury of the few who travelled frequently outside of the village.

The ICT resource centre, run by Myrada, is a community hub that hosts many self help group meetings, and provides IT courses for the community. This was the obvious choice of venue for our base, both for the social functionality and the technical infrastructure available. The staff has embraced the project and has proved to be an extra valuable resource.

\subsection{The StoryBank Concept}

StoryBank is inspired by the digital storytelling movement which has demonstrated the power of short two-minute audiovisual stories for compelling communication and empowerment within local communities in the west [1] [2]. This audiovisual format seemed to us to be ideal for giving a voice to those in the developing world who are disenfranchised from self-expression, internet use and other forms of written information sharing because they cannot read or write.

Hence one aim of the project is to make audiovisual story creation and sharing accessible to poor rural communities, and to test its value for empowerment and information sharing. What we have in mind is a kind of YouTube system for development, which extends initiatives that already provide local internet information in village ICT centers [5].

\section{THE STORYBANK INTERFACE 3.1 Initial Prototype}

From the very first discussions about the interface it was clear that we would opt for the completely visual option. The reason behind this was the high level of illiteracy in the area, and the variety of languages. As we are trying to look at the bigger picture, that at some point more villages, further afield could 
use this system, audio tagging would be of no benefit, as there would have to be a language translation for different regions. Additionally, users from the west should be able to interact with the system, as one of the aims of the project is to give us an insight to the needs and lives of the developing rural communities.

Our choice of device to capture and edit stories was mobile camera phones. The reason being, the versatility of the technology both to capture the story and to communicate it. Originally Multimedia Short Messaging (MMS) was the chosen technology, but after our first trip to the village, this was reconsidered as there was no GSM coverage. Additionally we found out that MMS is in general very problematic and not supported in all regions of India. This seemed to be a problem originally but, made us think of other alternative solutions, using WiFi and Bluetooth. Another observation that made us rethink our approach was the sense of community. When we visited the village the ICT resource centre staff showed us around and talked about the functions of the centre. Throughout our visit a swarm of people passed by, whether to find out about the next meeting of their self help group, or to assist in making a radio program, or attend an IT class, or just to say hello and see who we were. The building consists of two floors, with an office/ meeting room downstairs and upstairs a large patio, and three small rooms, one is a studio, one the editing room and the small IT classroom. The patio has a blackboard on the wall and is used for meetings and gatherings of various kinds. Essentially this area is the community hub. The buzz that was created by our visit brought on the idea of a situated display that would make the content of the StoryBank repository accessible to the community.

With all these observations and considerations we designed the first version of the system. The main purpose of this design was to provide a technological probe, and an interface to use in the second trip. A Nokia N80 camera phone with a very basic demo of the system, and a laptop with a wall mounted touch screen with an interface to access the repository which was populated with a number of existing radio programs and photographs from the village were taken to the village on the second trip. We present in the form of a case study the use of the camera phone and other prototyping artifacts as probes to engage the community in discussions on the design of the interface . Lessons learned and Future work which has been informed by this study are also discussed.

\subsection{Using the Prototype as a design probe}

The second trip took place late January early February 2007. Our experiences in the village resembled those described by [6]. We had a bit of a slow start which was a good reflection of the laid back Indian life. The setup of the situated display was delayed due to the power cuts, giving us a day to interact with the local community in a more informal way. Once the touch screen was up and running it instantly became a point of interest for the community as shown in figure 1 .

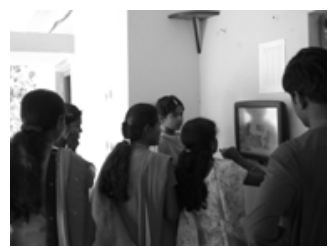

Figure 1 Community interacting with situated display
As it was constitution day and various activities were taking place in the village, the ICT resource centre was buzzing with visitors. The Manager who is very cooperative was proud to be involved with the project and that we were there to design with the community for the community the StoryBank interface. When the visitors left he gathered the resource centre and Namma Dhwani staff to be our first focus group. This meeting was important in formulating our methodology. The resource centre manager gave an introduction about the project and its importance for the village. After the introduction they were presented with the situated display interface. At this very early stage it became evident, that the icons that had been chosen for the story categories were not identified within the Indian culture. This was an issue that was anticipated; however their function was very important, as described in 3.2.1. The discussion of the camera phone interface and interaction followed. From the observation of how the focus group received the situated display interface, and their questions, a number of last minute changes were made to the methodology of these sessions. Additionally, it was decided that the focus groups would first have the session on the camera phone, which would be followed by the use of the touch screen. The main reason was that it was easier to explain the content and the interaction in the camera phone session. Over the 7 days that the study took place 8 focus groups were held in formal sessions. As these sessions took place on the patio, the number of participants increased, as friends of the participants who were passing by came up to see what was happening. These focus groups had 4-15 people and were of various educational levels, castes and financial status. As the sessions took place in the ICT resource centre, it was easy to have groups from various castes, as this is considered to be a neutral ground. The groups consisted of: resource centre and Namma Dhwani staff, farmers, women, Health workers, the a small group of people who speak English and are regarded to have a higher education than the others and school children.

\subsubsection{Methodology}

The focus group sessions were carried out on the patio area which had become our office space, as shown in figure 2, as we had access to electrical power from the UPS (Uninterrupted Power Supply) in the lab and radio station, and was visible to all the community, which as explained later on is also influenced our study. Our local researcher, who speaks all three languages of the village, and therefore can understand the local dialect, was the interpreter and cultural advisor in these sessions. The Interaction designer was the main facilitator. The same methodology was followed in all focus groups. With the exception of some of the radio station staff, most participants were not familiar with digital stories. The full understanding of digital stories was essential both for the interaction and design feedback from these sessions, but mainly to engage the community with the project by considering potential uses. The first day of our visit we prepared two photo narrative digital stories, one about the interaction designer, and one about a local child. The first consists of 5 photographs and 35 seconds of narrative in English. The second was made from photographs from three pictures that a local child drew about his home and favorite things, and a 44 second voice recording in the local dialect talking about them. These two simple stories were used to demonstrate the simplicity and versatility of digital stories. 


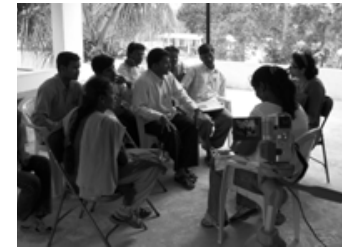

Figure 2 Focus group session

The sessions would typically start with a brief introduction about the project, followed by an introduction to digital storytelling, and the showing of the two stories. This prompted discussions of the potential stories that the participants could create. For example, the health workers immediately found great potential in making health education stories, which due to their small duration (maximum length 2 minutes) would not be tiring for the audience and would be simple and easy to create. Once the scope of the project was clear it was much easier to discuss the interface and the interaction. This was broken into two main parts: Icon identification and Interaction.

Icon identification. In the early stage of the first prototype design the Indian partners pointed out that only computer literate users would understand some of the icons we were planning to use. This is very much in line with observations made by G. Marsden in South Africa [3], [4]. The use of a set of icons to provoke a discussion and bring suggestion was agreed on, but due to the Indian culture it was stressed that the participants might be too shy to tell us their opinion or to disagree with our choice. One option to overcome this problem was to ask the participants to draw images that they thought would be functional was considered. This was almost immediately dismissed as too time consuming and could possibly limit the number of participants, as they could feel intimidated if their drawing skills were not particularly good. Icon identification seemed to be the obvious choice. Each of the icons used was presented full screen on a laptop. These were all clipart images, widely available, and considered to be easily identifiable in the European culture. Figure 3 shows the original icon set.

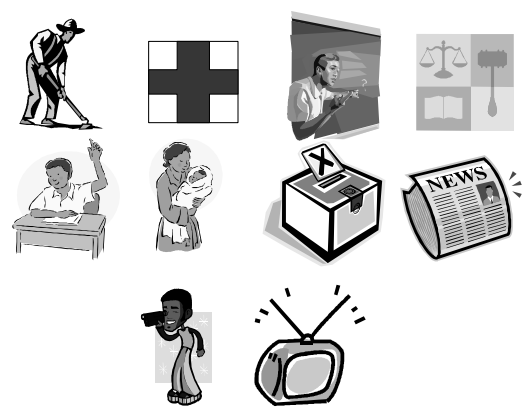

Figure 3 Original Icon set

These icons represented the story categories and user groupings. The last two icons were functional icons, for story capture and viewing. The participants were presented with the icon and asked to identify what it represented. At this point there was usually silence especially with the first icon that represented agriculture. The majority of the participants thought it was a man cleaning the street. They pointed out that farmers in India do not wear hats, but tie a cloth on their head, and that they wear a specific outfit which is easily identifiable. One of the participants drew a sketch of a farmer, which the others immediately recognized. In all focus groups this proved to be the least identifiable icon. The icon identification proved to be very successful as it was a good ice-breaker, giving the participants the opportunity to say their opinion without the danger of offending us, which was one of the cultural issues. The community had embraced us and did not want to appear to be disrespectful, consequently they would not say that they did not like something if asked their opinion. But identifying an icon was completely different as there was no issue of agreeing with us or offending us.

The discussions that sprung from this exercise gave us a clear picture of what the community preferences were. The main comment was that all icons had to relate with the rural Indian life style. For example, the news icon should be a person reading a local newspaper, the education a female teacher in a sari, with a ruler in her hand in front of a black blackboard with letters of the Kannada alphabet. The school children category icon, although identifiable, would be preferred to show a child in local school uniform. It was interesting to see that even though some icons were more easily identified, the participants would ask them to be more culture specific. By the time that the third icon was discussed the participants had overcome their original inhibitions and voiced freely their opinions. The Interaction designer had begun to understand the body language displayed by the participants and even though there was a strong language barrier, she could get the jest of the discussions taking place between the participants and the Indian researcher. There were times that the participants would get carried away and ask for great detail in the icons. That is where the N80 with the prototype came in to play, as by showing them the original icons on the phone they got an understanding of the size and detail that the icons would originally take. It is no surprise that this level of detail was requested by the community, as this is the first time that most of the participants will interact with a system through an icon representation, unlike western cultures where using a metaphor, or even a dead metaphor in some cases (such as the desktop) is the norm. From the outcome of these discussions a new set of icons was later developed, as shown in figure 4.

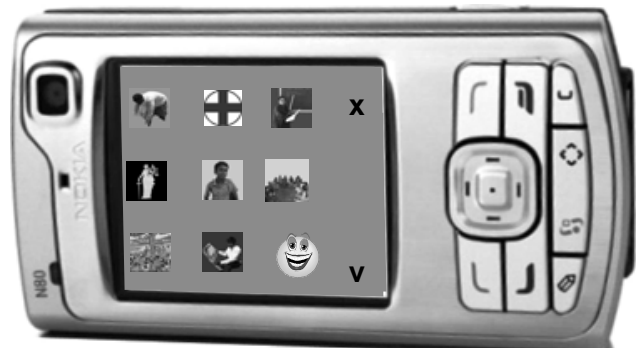

Figure 4 New Icon set

Interaction For this part of the discussion a sequence of cards with the suggested interface was used. The interface was described by the interaction designer and the cards were distributed among the participants. Each card was a cut out of the camera phone with the interface which was discussed at the moment at a $130 \%$ scale (figure 5). At the same time the interaction designer had the same interface on the laptop and went through the interaction process, while the Indian researcher translated using the actual camera phone as reference. As the semantic issues had been resolved this part of the conversations evolved around the actual interaction and the use of the interface. The original design had a log on screen, where the user was required to identify what group of user he or she belong to. These groups were identical to the story categories. The participants found this step just an extra burden, 
and of no relevance as there were various overlappings, as for example a child could also be a farmer. They also commented that the least possible steps would be preferable. Additionally, the community asked for a category on self help groups to be added Overall the interface was well accepted and the interaction understood and was picked up with minimum training.

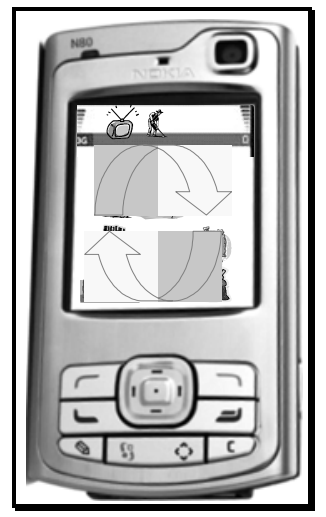

Figure 5 - Interface mock up card

Using the camera phone as a probe, even though the prototype was not fully functional, gave a good insight to the participants of how the system would function. Most of the participants had not used a mobile phone previously, and found the interaction straightforward, with the exception of the login screen. The participants also suggested that the navigation inside the application to capture or view a story. These suggestions were all taken on board and resulted in a complete design overhaul, where the capture, view and transfer functions are separate applications.

\section{DISCUSSION}

This part of the StoryBank project informed the design of the interface that will be tested and evaluated in the late summer. Additionally the experience from these focus groups and how they were held has helped us plan the testing and evaluation.

\subsection{Lessons Learnt}

Although the work carried out in this phase of the project seems to be very practical some very interesting research lessons were learn:

Work in open groups: this allowed people to join in the focus group activities when they felt comfortable, by first observing from a distance or getting reassurance from their peers that it was interesting and worth their time.

Use of combination of Hardware and Software props: As most of the community has not used mobile phones previously the combination of the camera phone to demonstrate the appearance of application with the sample stories and more detailed interface on the laptop gave the community a more concrete understanding of the system, resulting in discussions that formulated the current design. Additionally the card cut outs allowed the participants to envision how they could possibly interact with the device, removing the fear of damaging the device itself.

Finding ways of opening up creativity: Using open ended questions, discovery and recognition, made the participants to use their creativity. By using the icon identification method, the participants felt at ease and engaged with the creative part of the exercise, suggesting alternative icons.

\section{FUTURE/CURRENT WORK}

Since this study was carried out content generation activities have been run by the Indian researcher and the community. We have gathered about 20 stories dealing with village life, its people and the problems that they face in their every day lives. The StoryBank application has been redesigned and is on schedule to be tested and evaluated in the end of the summer.

\section{CONCLUSION}

The StoryBank project aims to provide a tool for a rural Indian community which will enable them to disseminate stories of their everyday life and experiences. Additionally it can be a powerful educational tool, which due to its portability can be used to take the stories to remote locations, with no power supply. The local community has embraced the project, and its participation has driven the design. At the time of writing, the revised design is being developed and its evaluation is planned to be carried out in the summer.

\section{ACKNOWLEDGMENTS}

The StoryBank project is funded by the UK Engineering and Physical Sciences Research Council. Staff includes David Frohlich, Dorothy Rachovides, Matt Jones, Will Harwood, Eran Edirisinghe, Dhamikke Wickramanayake, Mounia Lalmas, Paul Palmer, Arthur Williams, Roger Tucker, Ram Bhat and Maxine Frank.

\section{REFERENCES}

[1] BBC Capture Wales: Digital Storytelling http://www.bbc.co.uk/wales/capturewales/

[2] Lambert J. Digital Storytelling. Capturing lives: Creating communities. Digital Diner Press, 2003.

[3] Marsden G. Mobile Blog - Literacy http://web.mac.com/hciguy/iWeb/udev/Under\%20Develop ment/D8958C04-257E-414C-849B-A61F6E43C92B.html

[4] Marsden, G. "Using HCI to leverage communication technology” Interactions, Volume 10(2), ACM press, pp.48-55, 2003.

[5] OKN: http://www.openknowledge.net/

[6] Ramachandran, D., Kam , M.,Chiu, J., Canny, J., and Frankel J. L., Social Dynamics of Early Stage Co-Design in Developing Regions. In Proceedings of the SIGCHI conference on Human factors in computing systems (CHI '07) (San Jose, California, USA, April 28-may 3, 2007). ACM Press, New York, NY, 2007. 